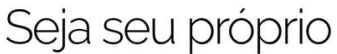 Diferencial
}
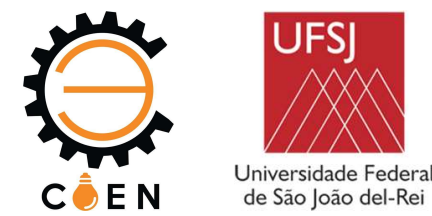

\section{PRODUÇÃO DE CIMENTO GEOPOLIMÉRICO A PARTIR DE CINZA VOLANTE E SOLUÇÕES ATIVADORAS SIMPLES E COMPOSTAS}

\author{
Adriano Galvão Souza Azevedo (1) (adrianogalvao26@yahoo.com.br), \\ Luis Fernando Tonholo Domingos (2) (luistonholo@gmail.com), Kurt Strecker (3) (strecker@ufsj.edu.br) \\ (1) Universidade Federal de São João del Rei (UFSJ) - DCNat - Praça Dom Helvécio, 74 - Fábricas - São João del Rei - MG \\ (2) Universidade Federal de São João del Rei (UFSJ) - DCNat - Praça Dom Helvécio, 74 - Fábricas - São João del Rei - MG \\ (3) Universidade Federal de São João del Rei (UFSJ) - DEMEC - Praça Frei Orlando, 170 - Centro - São João del Rei - MG
}

RESUMO: A indústria da construção civil está entre os setores que mais degradam o meio ambiente. Os geopolimeros são uma nova classe de material aglutinante capaz de ser utilizado como um substituinte para o cimento Portland comum. O objetivo deste trabalho foi avaliar as propriedades dos geopolímeros à base de cinzas volantes. As amostras geopoliméricas à base de cinzas volantes foram produzidas por ativação usando diferentes soluções alcalinas. Os resultados mostraram que a ativação com solução alcalina simples (somente $\mathrm{NaOH})$ promove alta resistência mecânica nas primeiras 24 horas $(88,0 \mathrm{MPa}$ ). $\mathrm{O}$ uso de silicato de sódio (solução de composto $\mathrm{NaOH}+\mathrm{Na} 2 \mathrm{SiO} 3$ ) promove um ganho de resistência mecânica próximo de $220 \%$ em algumas amostras. Após 28 dias de cura, as amostras produzidas com soluções ativadoras compostas apresentaram uma resistência à compressão de 50,8 MPa e 3,36\% de porosidade. A difração de raios X e a espectroscopia de infravermelho mostraram a presença das fases cristalinas e o deslocamento da banda de Si-O-Si existente na cinza volante original antes da ativação, respectivamente. A microscopia eletrônica de varredura mostrou que a morfologia esférica original da cinza volante foi modificada para uma superfície densificada e compactada devido à formação de aluminosilicato que aumentam a resistência mecânica.

PALAVRAS-CHAVE: Geopolímeros, cimento geopolimérico, cinza volante, ativação alcalina.

\section{INTRODUÇÃO}

Sabe-se que a produção de cimento Portland é um processo que consome enorme quantidade de energia, e também que sua produção é responsável por emissões de $\mathrm{CO} 2 \mathrm{em}$ cerca de $7 \%$ na atmosfera (ATIS et al., 2015).

Uma saída para o problema ambiental causado pela produção do cimento Portland e também para o consumo energético atrelado a sua produção é a utilização de um novo sistema de ligantes. A produção de cimentos álcali ativados se apresenta como um grande candidato a substituir o cimento Portland nas mais diversas áreas da construção civil (ATIS et al., 2015). Essa nova classe de ligantes, que é conhecida também como sendo polímeros inorgânicos ou Geopolímeros foi descrita pela primeira vez por Glukhovsky em 1959, ano em que a teoria base da ativação alcalina se desenvolveu. Em meados da década de 70 o nome geopolímero foi pela primeira vez citado por Joseph Davidovits. (PACHECO-TORGAL, F. et al., 2012, PACHECO-TORGAL e JALALI, 2007).

Joseph Davidovits junto com a sua equipe, desenvolveu um ligante geopolimérico, patenteado por ele na década de 1980 e como um recente produto tecnológico para aplicação estrutural - nas mais diversas áreas, que vão desde a indústria da construção civil, artística e até aplicações em setores da engenharia aeroespacial (BITENCOURT et al., 2012).

Os geopolímeros apresentam-se como sendo materiais inovadores e grandes candidatos a substituírem o cimento Portland devido, principalmente, suas baixas emissões de poluentes e sua superior durabilidade (DAVIDOVITS, 1988a).

Os geopolímeros podem ser produzidos em três unidades denominadas sialato, que dependem da relação atômica entre os átomos constituintes, conforme é mostrado na tabela abaixo. E a partir da 

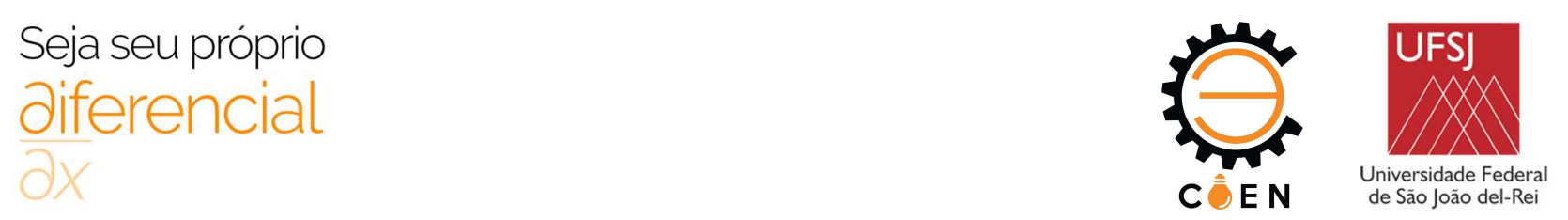

modificação da relação de Si:Al é possível obter materiais resultantes com diferentes propriedades físicas e mecânicas (DAVIDOVITS, 1991b, RAHIER e MELE e WASTIELS, 1996, BARBOSA e MACKENZIE e THAUMATURGO, 2000).

A construção civil tem sido o setor mais visado para o emprego dos geopolímeros, uma vez que suas propriedades podem ser muito similares, ou até mesmo superiores, às dos materiais cerâmicos aplicados nesse campo. No entanto, o maior diferencial dos geopolímeros em relação a esses produtos convencionais está em seus aspectos relacionados à sustentabilidade. O processo de geopolimerização necessita de um menor consumo energético pode ser realizado pelo uso de subprodutos industriais (NATH et al., 2016). Em relação ao consumo de energia e a emissão de gases de estufa, o cimento geopolimérico emite $80 \%$ menos de CO2 e consome $30-40 \%$ menos de energia do que o cimento Portland, em sua produção (YAHYA et al., 2017).

Nos dias atuais subprodutos são utilizados como aditivos minerais (cinzas volantes ou escória granulada de alto forno) em concretos como substituição do cimento. Na fase de endurecimento, as propriedades do geopolímero são influenciadas pela utilização de cinzas volantes, que melhora a trabalhabilidade, aumenta a resistência mecânica, reduz a contração e o aumento de temperatura aumenta de cura promove uma maior resistência à abrasão. Além disso, a utilização da cinza como material de partida na produção dos geopolímeros minimizam os problemas de armazenamento e possível contaminação do ar com materiais particulados (RAHIER e MELE e WASTIELS, 1996).

O desenvolvimento da microestrutura geopolimérica é dependente das características das matérias-primas e de parâmetros do processo, especialmente da concentração alcalina da solução ativadora utilizada e da temperatura de cura (YAHYA et al., 2017). Estudos têm demonstrado que a temperatura de cura é um parâmetro que tem papel importante no processo geopolimerização (NATH et al., 2016).

À temperatura ambiente, o processo de geopolimerização em geopolímeros a base de cinza volante possui baixa efetividade, apresentando um aumento na cinética de formação da rede geopolimérica com o aumento da temperatura de cura, principalmente nas primeiras horas de síntese (ATIS et al., 2015, AZEVEDO, A.G.S. et al., 2017, GÖRHAN e KÜRKLÜ, 2014).

De acordo com Davidovits et al. (1985), a policondensação dos íons a base de silício e alumínio está diretamente ligada ao tempo e temperatura da cura térmica (DAVIDOVITS, J. e SAWYER, 1985). Os dados apresentados pelos autores descrevem que a policondensação ocorre dentro de 15 horas a temperatura ambiente $\left(\approx 22{ }^{\circ} \mathrm{C}\right)$. Entretanto, submetendo o mesmo material a cura de 50,85 e $90{ }^{\circ} \mathrm{C}$ os autores concluíram que as reações são aceleradas para períodos próximos de 4, 1,5 e 0,5 horas, respectivamente. Esses resultados demonstram que para aplicações onde são necessárias maiores resistências iniciais os geopolímeros se apresentam como bons substitutos aos cimentos tradicionais. Vale ressaltar que as reações podem ser aceleradas por meio de outras técnicas, tais como: micro-ondas, cura em altas frequências e sob efeito Joule, colocando fios elétricos em contato com a pasta fresca (DAVIDOVITS, J. e SAWYER, 1985).

Com o intuito de se obter matrizes de elevada resistência à compressão e flexão Görür et al. (2015) avaliaram a produção de geopolímeros fabricados com cinzas volantes contendo baixos teores de cálcio (tipo F). Os autores submeteram os corpos de prova fabricados com diferentes concentrações de $\mathrm{Na} 2 \mathrm{O}$ a elevadas temperaturas e diferentes tempos de cura, $45-115^{\circ} \mathrm{C}$ e 24,48 e 72 horas respectivamente. Os autores demonstraram que é possível obter matrizes geopoliméricas com resistências à compressão e flexão de 120 e $15 \mathrm{MPa}$, respectivamente utilizando solução ativadora com $14 \% \mathrm{NaOH}$ e cura térmica de 24 horas a $115^{\circ} \mathrm{C}$. Eles concluíram que é possível 


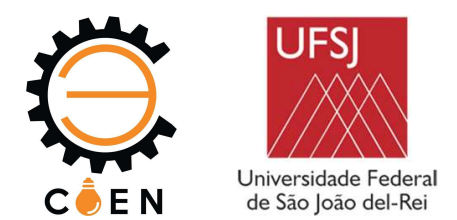

controlar os valores de resistência modificando a concentração do ativador, tempo e temperatura de cura, obtendo ao final do processo materiais que atendam as mais diversas áreas da construção civil como substituinte do cimento Portland comum, o que também foi encontrado no trabalho de (AZEVEDO, A G S e STRECKER, 2017a).

O intuito deste trabalho é fazer um complemento aos resultados apresentados por Azevedo et al., onde os autores produziram geopolímero que atingem 38,5 $\pm 1,5 \mathrm{MPa}$ de resistência à compressão e $27,2 \%$ de porosidade aparente (AZEVEDO, A G S e STRECKER, 2017a, AZEVEDO, A G S e STRECKER, 2017b). Neste trabalho, foi analisada a influência do aumento de temperatura de cura dos corpos de prova, sendo utilizada a temperatura de $90{ }^{\circ} \mathrm{C}$, e a variação na concentração das soluções de $\mathrm{NaOH}$ (12 e $16 \mathrm{M}$ - aumento da \% de Na2O) na produção dos geopolímeros, mantendo paramentos como tempo de cura e dimensão dos corpos de prova e seguindo os procedimentos realizado pelos autores.

\section{MATERIAIS E MÉTODOS}

\subsection{Materiais}

\subsubsection{Cinza Volante}

Como fonte de aluminossilicato utilizou-se a cinza volante proveniente do complexo termelétrico de Jorge Lacerda/SC. As cinzas são distribuídas pela empresa PozoFly - comércio de cinzas LDTA. A cinza recebida na forma bruta é moída em moinhos de bolas e ensacada para posterior distribuição.

\subsubsection{Hidróxido de Sódio $(\mathrm{NaOH})$}

Para a confecção das soluções ativadoras se utilizou um hidróxido de sódio da marca Sulfal Química com pureza de $98 \%$ na forma de escamas.

\subsubsection{Silicato de Sódio (Na2SiO3)}

O silicato de sódio utilizado para o fornecimento de $\mathrm{SiO} 2$ para a solução foi obtido da empresa Diatom ${ }^{\circledR}$. Na Tabela 2 é observada a composição química e as propriedades físicas apresentadas pelo silicato fornecidas pelo fabricante.

\subsubsection{Métodos}

Para a produção das amostras dos geopolímeros as diferentes soluções, listadas na Tabela, foram misturadas com a cinza volante previamente peneirada para a remoção de partículas grosseiras. A pasta foi produzida pela mistura da cinza com as soluções com auxílio de um misturador mecânico por 5 minutos na temperatura ambiente.

As pastas frescas foram posteriormente colocadas em moldes com dimensões de 50 x $25 \mathrm{~mm}$ (altura x largura), submetidas à vibração para a remoção das bolhas e melhor acomodação da pasta dentro dos moldes e seladas com filme plástico para evitar perda de água por evaporação. As amostras foram curadas em temperatura ambiente por 24 horas. A cura térmica foi realizada pela remoção dos filmes plásticos e acomodação dos moldes em estufa por 24 horas a $90^{\circ} \mathrm{C}$. As amostras foram curadas por mais 1,7 e 28 dias em temperatura laboratorial antes da realização das caracterizações das propriedades mecânicas, químicas e físicas dos geopolímeros a base de CV. 

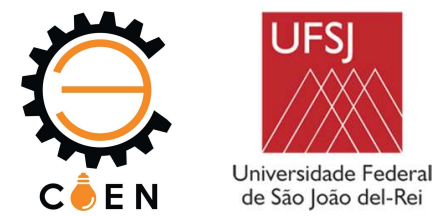

A nomenclatura utilizada para se identificar as amostras consiste em apresentar a concentração de $\mathrm{NaOH}$ (mol.L-1) das soluções ativadoras, dada pela letra $\mathrm{H}$ e também a porcentagem de $\mathrm{Na} 2 \mathrm{SiO} 3$ utilizada em cada mistura (\% em massa), que é representada pela letra $\mathrm{S}$. O tempo de cura é expresso pelo número de dias de cura, logo a frente da composição da solução ativadora utilizada. Após o número que indica o tempo de cura, é colocada temperatura de cura das amostras. Como exemplo podemos citar a amostra H16S20-7-90 que corresponde a amostra produzida utilizando a solução de $\mathrm{NaOH}(16 \mathrm{M})$ e com $20 \%$ de Na2SiO3 após 7 dias de cura, levada a estufa à temperatura de $90^{\circ} \mathrm{C}$.

Tabela 1. Informações acerca das soluções alcalinas utilizadas para produzir os geopolímeros a base de cinza volante. (CV) cinza volante, (SA) solução ativadora.

\begin{tabular}{ccccccc}
\hline Amostra & $\begin{array}{c}{[\mathbf{N a O H}]} \\
\text { mol.L }^{-1}\end{array}$ & \%Na2SiO$_{3}{ }^{\mathbf{b}}$ & $\mathbf{\% N a}_{2} \mathbf{O}^{\mathbf{a}}$ & $\mathbf{\% S i O}_{2}{ }^{\mathbf{a}}$ & $\mathbf{S i O}_{2} / \mathbf{N a}_{2} \mathbf{O}^{\mathbf{a}}$ & $\begin{array}{c}\mathbf{H}_{2} \mathbf{O} / \mathbf{C V} \\
\text { (massa) }\end{array}$ \\
\hline H12S0 & 12 & 0 & 13,51 & 0 & 0 & 0,33 \\
H16S0 & 16 & 0 & 16,98 & 0 & 0 & 0,28 \\
H12S10 & 12 & 10 & 12,31 & 3,36 & 0,27 & 0,31 \\
H16S10 & 16 & 10 & 15,08 & 3,36 & 0,22 & 0,28 \\
H12S20 & 12 & 20 & 11,11 & 6,71 & 0,60 & 0,30 \\
H16S20 & 16 & 20 & 13,18 & 6,71 & 0,51 & 0,27 \\
H12S30 & 12 & 30 & 9,90 & 10,07 & 1,02 & 0,28 \\
H16S30 & 16 & 30 & 11,29 & 10,07 & 0,89 & 0,27 \\
\hline
\end{tabular}

a em relação à quantidade de cinza volante adicionada.

b em relação à quantidade total de solução (em massa).

Um total de 10 corpos de prova de cada condição foram utilizados para as medidas das resistências à compressão. Para as análises microestruturais foram utilizados fragmentos dos corpos de prova fraturados. Os mesmos foram quebrados, macerados em moinho de ágata e peneirados em peneira de \#100 Mesh para as análises microestruturais. As amostras foram caracterizadas por meio da compressão uniaxial como descrito na norma NBR - 5739 (ABNT, 2007), usando uma máquina universal da marca Shimadzu modelo AG-X plus. Os dados obtidos foram tratados por meio da utilização do software Trapezium X - versão 1.2.6 - Shimadzu ${ }^{\circledR}$.

As cinzas volantes foram caracterizadas utilizando um equipamento da marca Shimadzu Energy Dispersive X-ray Fluorescence Spectrometer, modelo EDX 8000 para a obtenção da composição química da cinza volante. A massa específica da cinza volante utilizada nesse trabalho foi obtida por um picnômetro a Hélio da marca Micromeritics, modelo AccuPyc 1340. A granulometria foi obtida utilizando um aparelho da marca Malvern modelo Mastersizer 2000 (0.02 $2000 \mu \mathrm{m})$. As amostras de cinzas foram dispersas em água deionizada e as condições de ensaio foram: agitação de 1500 RPM, tempo de ultrassom de 2,5 min, obscuração entre 10 e 20\% e tempo de dispersão de 5 min.

A caracterização das fases cristalinas da cinza volante e das pastas álcali ativadas foi realizada por meio da utilização de um difratômetro da marca Shimadzu modelo XRD-6000, com voltagem de 


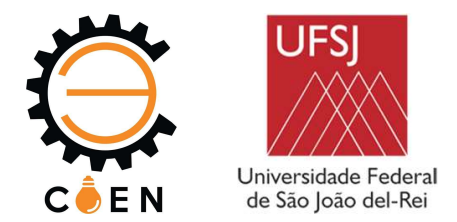

$30 \mathrm{kV}$, corrente de $30 \mathrm{~mA}$, radiação utilizada foi $\mathrm{Cu}-\mathrm{K} \alpha(\lambda=0,15462 \mathrm{~nm})$ com passo de $0,02^{\circ} \mathrm{e}$ velocidade de varredura de $2 \% \mathrm{mim}$, entre 5 e $60^{\circ}$. A identificação das fases foi realizada por comparação dos picos de difração obtidos com os fornecidos pelos cartões do ICPDS ("Inorganic Crystal Structure Database" - Banco de Dados de Estruturas Cristalinas Inorgânicas).

Para a análise da morfologia das amostras de cinza volante e dos geopolímeros foi utilizado um microscópio eletrônico da marca Hitachi, modelo TM 300, com EDS acoplado da marca Bruker, modelo X-Flash mim SVE. As propriedades físicas dos geopolímeros foram obtidas utilizando a norma Britânica BS EN ISO 10545-3 (1997). Os valores de permeabilidade dos diferentes geopolímeros foram calculados como descrito no trabalho de Miyake, (1994).

\section{RESULTADOS E DISCUSSÃO}

\subsection{Caracterização da Cinza Volante}

\subsubsection{Fluorescência de Raios X (FRX)}

A CV é composta basicamente de sílica e alumina. Entretanto, existe a presença de outros elementos em menores quantidades, como apresentado na Tabela 2. A cinza volante é do tipo F, de acordo com a norma ASTM C618 (2010) que classifica como cinzas volantes desse tipo o material que apresenta um teor de $\mathrm{SiO} 2, \mathrm{~A} 12 \mathrm{O} 3$ e Fe2O3 maior do que $70 \%$.

Tabela 2. Composição química da cinza volante utilizada para a produção de geopolímeros.

\begin{tabular}{ccccccccccc} 
SiO2 & Al2O3 & Fe2O3 & K2O & CaO & TiO2 & SO3 & ZrO2 & V2O5 & Outros Óxidos* & SiO2/Al2O3 \\
\hline 56.025 & 29.406 & 6.155 & 3.771 & 2.069 & 1.805 & 0.255 & 0.114 & 0.104 & 0,769 & 3,88 \\
\hline
\end{tabular}

*( $\mathrm{ZnO}, \mathrm{MnO}, \mathrm{Cr}_{2} \mathrm{O}_{3}, \mathrm{SrO}, \mathrm{CuO}, \mathrm{Rb}_{2} \mathrm{O}, \mathrm{Y}_{2} \mathrm{O}_{3}, \mathrm{PbO}, \mathrm{Ga}_{2} \mathrm{O} 3, \mathrm{GeO} 2, \mathrm{NiO}$ e $\left.\mathrm{NbO}\right)$

\subsubsection{Análise Granulométrica a Laser e Picnometria}

Na Tabela 3 são apresentados os valore de D10, D50 e D90 da CV utilizada na produção dos corpos de prova. Os tamanhos das partículas variam entre $0,275 \mu \mathrm{m}$ e $209 \mu \mathrm{m}$. A massa específica da cinza volante é $2,21 \pm 0,01 \mathrm{~g} / \mathrm{cm}^{3}$.

Tabela 3. Diâmetros das partículas presentes na cinza volante (D90, D50 e D10) e massa específica.

\begin{tabular}{ccc}
\hline Diâmetro & Tamanho $(\boldsymbol{\mu m})$ & Massa específica $(\mathbf{g} / \mathbf{c m} 3)$ \\
\hline D10 & 4,99 & \\
D50 & 30,82 & $2,21 \pm 0,01$ \\
D90 & 115,12 & \\
\hline
\end{tabular}

\subsubsection{Difração De Raios X (DRX)}

O difratograma da cinza volante apresenta vários picos de difração de fases cristalinas que podem estar presentes no carvão ou podem se formar durante a queima dos mesmos nas usinas termelétricas (WILLIAMS e VAN RIESSEN, 2010). Picos de difração correspondentes a fases 


\section{Seja seu próprio aiferencial}

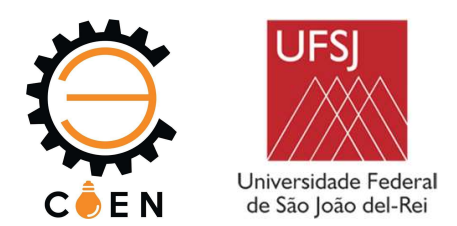

cristalinas de quartzo - $\mathrm{SiO}_{2}$ (ISCD\#100341), mulita - $\mathrm{Al}_{6} \mathrm{Si}_{2} \mathrm{O}_{13}$ (ISCD\#66447, \#66448, \#66449) e hematita - $\mathrm{Fe}_{2} \mathrm{O}_{3}$ (ISCD\#82137) foram identificados na CV. O difratograma da cinza também apresenta um halo entre 18 e $35^{\circ} 2 \theta$ que confirma a presença de material amorfo, na sua grande maioria os aluminossilicatos amorfos que irão reagir durante a ativação da cinza com as soluções alcalinas.

\subsubsection{Microscopia Eletrônica De Varredura (MEV)}

É possível notar que o material é composto de partículas esféricas e tamanho micrométrico. Possui tamanho aleatório, como apresentado nos resultados de granulometria. As esferas podem ser ocas ou podem conter partículas de menor dimensão dentro do seu interior (GUO e SHI e DICK, 2010, PROVIS e DEVENTER; S.J., 2009).
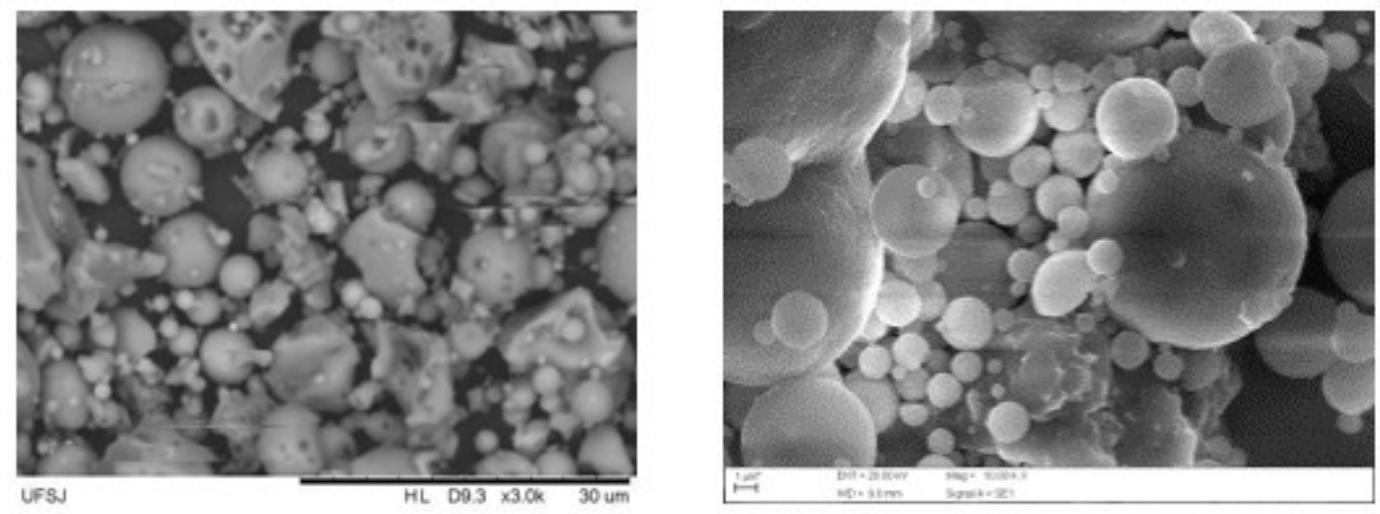

Figura 1. Microscopia eletrônica de varredura da CV volante utilizada para a produção dos geopolímeros.

\subsubsection{Resistência à Compressão}

Os valores da resistência à compressão dos geopolímeros enrijecidos após diferentes tempos de cura são apresentados na Tabela 4. As amostras ativadas com solução contendo apenas $\mathrm{NaOH}$ apresentaram os mais elevados valores de resistência à compressão. Para a amostra H12S0-28-90 o valor de $72 \mathrm{MPa}$ foi obtido após os 28 dias de cura. A amostra H16S0-1-90 apresentou uma resistência próxima de $88 \mathrm{MPa}$ Após as primeiras $24 \mathrm{~h}$ de cura, atingindo o mais alto valore de resistência à compressão dentre todas as amostras estudadas. Tal resultado está de acordo com o trabalho de Görür et al. (2015), em que os autores obtiveram maiores valores de resistência à compressão nas amostras ativadas com elevados teores de $\mathrm{Na}_{2} \mathrm{O}$.

Tabela 4. Valores de resistência a compressão dos geopolímeros a base de cinza volante em diferentes tempos de cura.

\begin{tabular}{cccc}
\hline \multirow{2}{*}{ Amostra } & \multicolumn{3}{c}{ Resistência à compressão (MPa) } \\
\cline { 2 - 4 } & 1 Dia & 7 Dias & 28 Dias \\
\hline \multirow{2}{*}{ H12S0-90 } & $33,4 \pm 9,2$ & $36,1 \pm 6,2$ & $72,3 \pm 1,2$ \\
H16S0-90 & $88,0 \pm 8,0$ & $86,9 \pm 4,5$ & $71,1 \pm 9,0$
\end{tabular}

IX COEN - Congresso de Engenharias da UFSJ Interconexão. 


\begin{tabular}{llll} 
H12S10-90 & $33,9 \pm 6,2$ & $23,5 \pm 2,2$ & $24,8 \pm 3,4$ \\
H16S10-90 & $38,8 \pm 3,1$ & $32,4 \pm 2,6$ & $24,6 \pm 4,3$ \\
H12S20-90 & $46,2 \pm 5,0$ & $47,6 \pm 1,2$ & $46,9 \pm 4,6$ \\
H16S20-90 & $31,4 \pm 5,1$ & $51,6 \pm 6,5$ & $50,8 \pm 5,9$ \\
H12S30-90 & $59,1 \pm 5,1$ & $53,4 \pm 5,3$ & $60,5 \pm 5,2$ \\
H16S30-90 & $44,7 \pm 5,3$ & $34,4 \pm 3,4$ & $43,0 \pm 9,2$ \\
\hline
\end{tabular}

Outro ponto que deve ser ressaltado é que a maior concentração de $\mathrm{NaOH}$ utilizada nas soluções promove o aumento da temperatura de evaporação das moléculas de água presentes. Tal fato dificulta a evaporação das moléculas de água em temperaturas próximas de $90{ }^{\circ} \mathrm{C}$, o que favorece o transporte das espécies dissolvidas e consequentemente a formação do gel aluminossilicato responsável pelo ganho de resistência mecânica observado. É possível observar o decréscimo dos valores de resistência à compressão após 28 dias de cura. Tal fato pode estar relacionado com o excesso de álcali na rede geopolimérica, que pode se precipitar e provocar perturbações na estrutura geopolimérica.

Com o início do uso de $\mathrm{Na} 2 \mathrm{SiO} 3$ na composição das soluções ativadoras das amostras curadas à $90^{\circ} \mathrm{C}$, os valores de resistência apresentaram comportamento diferente das amostras produzidas com as soluções ativadoras simples (apenas $\mathrm{NaOH}$ ). As amostras H12S10-90 e H16S10-90 apresentaram um decréscimo na resistência após a adição de $10 \%$ de $\mathrm{Na} 2 \mathrm{SiO} 3$ na solução. A diminuição pode estar relacionada à redução de água presente nas amostras, o que desfavorece a geopolimerização, e também o fato da redução na concentração de $\mathrm{Na} 2 \mathrm{O}$, como apresentado na Tabela 1, fazendo com que o decréscimo na concentração de álcali diminua a dissolução das partículas de cinza volante. Deve-se ressaltar que as soluções foram ativadas com diferentes quantidades de $\mathrm{H} 2 \mathrm{O}$ e $\mathrm{Na} 2 \mathrm{O}$, provenientes tanto da solução de $\mathrm{NaOH}$ quanto da solução de $\mathrm{Na} 2 \mathrm{SiO} 3$. As moléculas de água, além de atuarem como lubrificantes das partículas de cinza volante ajudando na melhoria da trabalhabilidade das pastas, elas atuam como transportadoras de espécies após a dissolução. As diferentes pastas continham quantidades de água distintas, como mostrado na Tabela 1, o que pode ter afetado o processo de dissolução e consequentemente as reações de ganho de resistência mecânica (geopolimerização).

$\mathrm{O}$ aumento da quantidade de $\mathrm{SiO} 2$ na solução ativadora favorece a formação do geopolímero e consequentemente o aumento da resistência mecânica. As amostras H12S30-28-90 e H16S30-2890 apresentaram valores de resistência próximos de 60 e $43 \mathrm{MPa}$, respectivamente. O maior valor de resistência mecânica apresentado pela amostra com menor concentração de $\mathrm{Na} 2 \mathrm{O}$ pode estar relacionado com a combinação $\mathrm{NaOH} / \mathrm{Na} 2 \mathrm{SiO} 3$ que favorece a ocorrência da geopolimerização, como apresentado na Figura 2. Tais resultados estão de acordo com os resultados encontrados por Škvára et al. (2005), em que maiores valores de resistência à compressão foram obtidos quando a razão $\mathrm{SiO} 2 / \mathrm{Na} 2 \mathrm{O}$ da solução ativadora composta foi ajustado próximo de 1,0.

Analisando os resultados, podemos observar que as amostras que apresentaram elevadas concentrações de álcalis em sua composição (elevadas quantidades de $\mathrm{Na} 2 \mathrm{O}$ ) apresentaram um ganho de resistência mecânica pronunciado nos primeiros dias. Entretanto, passados os 28 dias de cura as amostras apresentam um decréscimo na resistência à compressão, como discutido anteriormente. Esses resultados estão de acordo com os encontrados no trabalho de Fernández-Jiménez e Palomo 


\section{Seja seu próprio Diferencial}

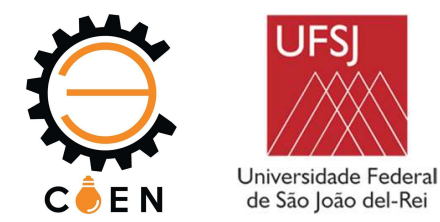

(2005). em que os autores obtiveram um maior valor de resistência à compressão na amostra contento aproximadamente $13,0 \%$ [Na2O] em relação à quantidade de cinza volante.

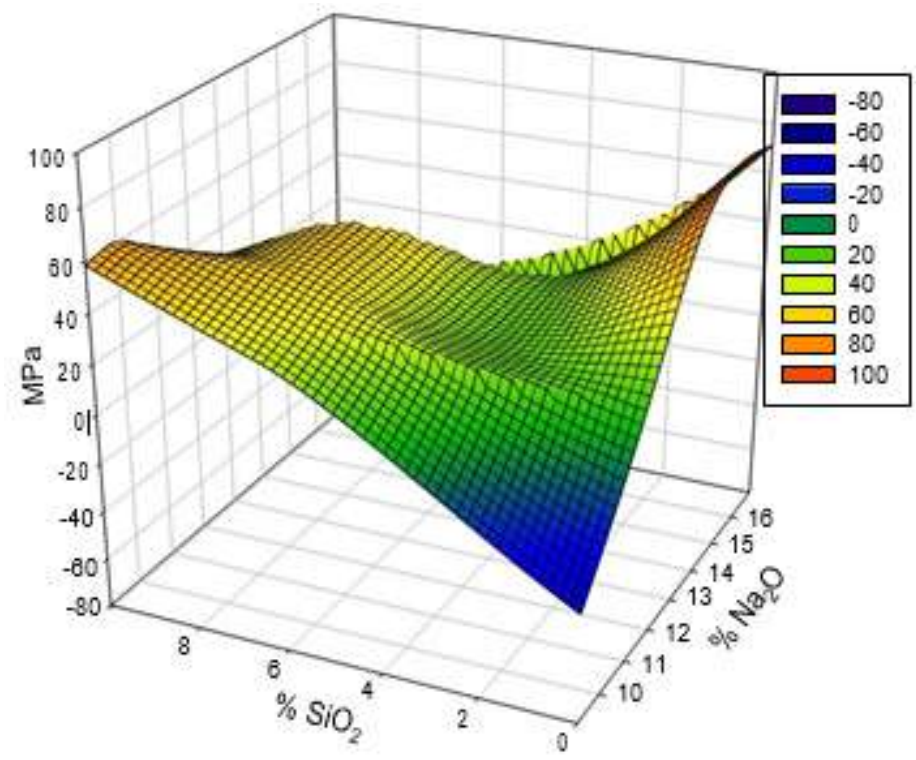

Figura 2. Influência da concentração de $\mathrm{SiO}_{2}$ e $\mathrm{Na}_{2} \mathrm{O}$ no ganho de resistência à compressão de geopolímeros a base de $\mathrm{CV}$ após 28 dias de cura.

\subsubsection{Absorção de Água, Porosidade Aparente, Densidade e Permeabilidade}

A Tabela mostra os resultados da análise de absorção de água, porosidade aparente, densidade e permeabilidade das amostras após 28 dias de cura. A amostra que apresentou maior absorção de água foi a H12S30, com aproximadamente $13 \%$. O teste de absorção de água está relacionado com a presença de porosidade aberta nos geopolímeros, sendo diretamente proporcional à absorção de moléculas de $\mathrm{H}_{2} \mathrm{O}$.

Os valores da densidade dos corpos de prova se apresentaram bem próximos ao da cinza volante utilizada na fabricação dos corpos de prova, com valores em torno de $2,0 \mathrm{~g} / \mathrm{cm}^{3}$. A adição de silicato de sódio promoveu uma diminuição da porosidade do material. Tal fato pode ser associado com a formação de uma estrutura mais densificada quando $\mathrm{SiO}_{2}$ livre é adicionado ao sistema pela utilização do $\mathrm{Na}_{2} \mathrm{SiO}_{3}$. As porosidades das amostras H12S10-28-90 e H12S30-28-90 foram de 16,7 e 19,03 \%, respectivamente. Esse aumento da quantidade de poros pode estar relacionado com a menor quantidade de água com o aumento da concentração de $\mathrm{Na}_{2} \mathrm{SiO}_{3}$, o que diminui a trabalhabilidade das pastas frescas e consequentemente promove a maior porosidade dos CP's durante o preenchimento dos moldes.

Tabela 5. Valores de absorção de água, porosidade aparente, densidade e permeabilidade dos corpos de prova após 28 dias de cura.

\begin{tabular}{ccccc}
\hline Amostra & $\begin{array}{c}\text { Absorção de } \\
\text { água (\%) }\end{array}$ & $\begin{array}{c}\text { Porosidade } \\
\text { aparente (\%) }\end{array}$ & Densidade $\left(\mathbf{g} / \mathbf{c m}^{\mathbf{3}}\right)$ & Permeabilidade $\left(\mathbf{E}^{\mathbf{- 1 6}} \mathbf{m}^{\mathbf{2}}\right)$ \\
\hline H12S0-28-90 & $11,41 \pm 0,1$ & $18,6 \pm 0,2$ & $2,0 \pm 0,01$ & $297,8 \pm 12,0$
\end{tabular}




\section{Seja seu próprio \\ Diferencial \\ ox}

\begin{tabular}{ccccc} 
H16S0-28-90 & $12,38 \pm 0,1$ & $20,01 \pm 0,2$ & $2,02 \pm 0,02$ & $115,34 \pm 0,7$ \\
H12S10-28-90 & $10,37 \pm 0,2$ & $16,7 \pm 0,3$ & $1,93 \pm 0,01$ & $70,91 \pm 7,7$ \\
H16S10-28-90 & $9,12 \pm 0,6$ & $15,3 \pm 0,9$ & $1,97 \pm 0,01$ & $58,68 \pm 2,4$ \\
H12S20-28-90 & $13,12 \pm 1,6$ & $19,2 \pm 4,6$ & $1,80 \pm 0,3$ & $85,46 \pm 51,2$ \\
H16S20-28-90 & $11,01 \pm 0,5$ & $18,02 \pm 0,8$ & $1,99 \pm 0,03$ & $124,22 \pm 16,2$ \\
H12S30-28-90 & $11,64 \pm 2,4$ & $19,03 \pm 3,5$ & $2,02 \pm 0,04$ & $136,32 \pm 85,5$ \\
H16S30-28-90 & $4,2 \pm 3,65$ & $9,11 \pm 6,80$ & $2,03 \pm 0,14$ & $154,81 \pm 12,1$ \\
\hline
\end{tabular}

A permeabilidade das amostras fabricadas somente com as soluções de $\mathrm{NaOH}$ tiveram uma permeabilidade mais elevada, 297,8 e 115,34 E-16 m2 para as amostras H12S0-28-90 e H16S0-2890, respectivamente. Esse maior valor pode estar relacionado com a maior quantidade de água presente nessas soluções, que, ao evaporarem, deixam pequenas cavidades que atuam aumentando a permeabilidade dos CP'S. Após a adição de silicato, a permeabilidade tem um decréscimo de 297 para 70,9 E-16 m2, comprovando que a adição de silicato promove o aparecimento de uma matriz com menor quantidade de poros interligados. A amostra H16S20-28-90 apresentou elevado valor para a permeabilidade, possivelmente causado por trincas e defeitos no material, devido, possivelmente, à sua baixa trabalhabilidade durante a produção dos corpos de prova geopoliméricos.

\subsubsection{Difração de Raios X (DRX)}

A Figura 3 apresenta os resultados da difração de raios $\mathrm{X}$ das amostras das cinzas volantes e dos geopolímeros produzidos com as diferentes soluções alcalinas e curados à $90{ }^{\circ} \mathrm{C}$. É possível observar características semelhantes nas fases cristalinas das amostras dos geopolímeros ativados com as soluções ativadoras distintas. As amostras apresentaram picos referentes às fases cristalinas presentes na cinza volante original. Isso demonstra que as fases de elevada ordenação atômica não participam do processo de geopolimerização devido sua baixa solubilidade nas soluções ativadoras. Entretanto, o halo correspondente à matriz de aluminossicatos amorfos presentes na cinza volante entre 18 e $35^{\circ} 2 \theta$ se desloca para angulações próximas de 18 e $40^{\circ} 2 \theta$, o que, de acordo com Guo et al. (2010), confirma a formação do gel aluminossilicato amorfo e que é o responsável pelo ganho de resistência mecânica da pasta geopolimérica. Com a adição do $\mathrm{Na} 2 \mathrm{SiO} 3$ no sistema é possível observar picos correspondentes à fase $\mathrm{Na}$ - Chabazita - NaAlSi2O6·3H2O (JCPDS - 19-1178). Esse composto é um precursor zeolítico muito identificado em processos de obtenção de zeólitas artificiais (CRIADO et al., 2008, BAKHAREV, 2005). Os picos de difração observados na cinza volante original apresentam uma variação nas suas intensidades. Tal observação foi relacionada com a formação do gel aluminossilicato amorfo, que pode criar uma cobertura em determinadas regiões do material, tornando difícil a identificação da fase cristalina. 

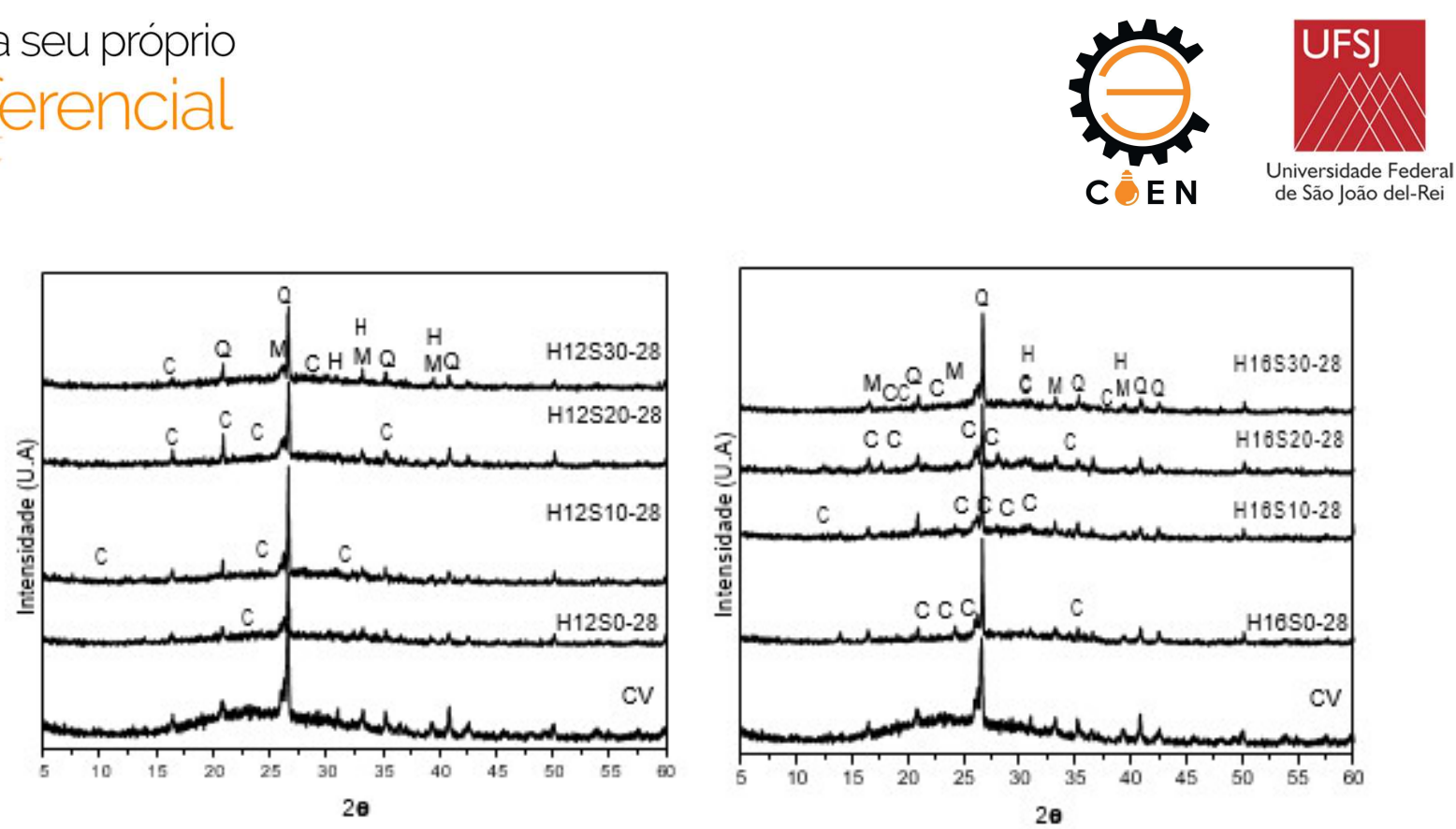

Figura 3. DRX da cinza volante original e das amostras de geopolímeros após 28 dias de cura, curadas a $90^{\circ} \mathrm{C}$.

\subsubsection{Microscopia Eletrônica de Varredura e Espectroscopia de Energia Dispersiva (MEV/EDS)}

A Figura 4 mostra as imagens superficiais dos geopolímeros produzidos com diferentes soluções ativadoras à temperatura de $90{ }^{\circ} \mathrm{C}$. É possível observar a presença de partículas esféricas envoltas pelo gel de aluminossilicato amorfo mesmo após o contato com as soluções ativadoras. Nas amostras à $90^{\circ} \mathrm{C}$, notou-se maior formação de uma matriz geopolimérica quando se utiliza a solução composta para a ativação da cinza volante. Nas amostras H12S10-28-90 e H16S10-28-90 é possível observar uma maior quantidade de matriz formada, com partículas embebidas na matriz. Tal fato demonstra que a diminuição da concentração de $\mathrm{Na} 2 \mathrm{O}$ nas soluções favorece o aparecimento de matrizes geopolimérica contendo partículas de CV intactas. As amostras H12S0-28-90 e H16S0-2890 apresentam uma superfície com elevada quantidade do gel aluminossilicato. Isso corrobora com os resultados de resistência à compressão em que essas amostras se apresentaram superiores.

$\mathrm{Na}$ Tabela 6 são apresentados os resultados das análises de EDS das amostras geopoliméricas. As redes tridimensionais encontradas nos geopolímeros podem ser representadas pela fórmula geral Rn-[[-Si-O-]z-Al-O]n.wH2O ( $\mathrm{R}$ representa um cátion tal como sódio, potássio ou cálcio); $\mathrm{n}$ representa o grau de policondensação; $Z=1,2$ ou 3 e W é a quantidade de água ou grau de hidratação do sistema. Nas amostras produzidas nesse trabalho foi possível observar que a razão $\mathrm{Si} / \mathrm{Al}$ das amostras se encontra entre valores próximos de 1,8 e 2,8, demonstrando que a estrutura formada durante a geopolimerização pode ser uma variação entre o Poli(sialato-siloxo) e Poli(sialatodisiloxo). A razão $\mathrm{Na} / \mathrm{Al}$ de acordo com a fórmula geral apresentada acima, deve estar o mais próximo possível de 1,0, visando à obtenção de uma estrutura eletricamente neutra. A amostra H16S0-90 apresentou o valor mais próximo de $1,0(0,99)$ para essa razão, demonstrando que a estrutura formada está perto do balanceamento completo. Essa configuração colabora para uma matriz mais estável com maior ganho de resistência mecânica, como é observado no ensaio de resistência mecânica.

Tabela 6. Análise de EDS das amostras de GP produzidas com diferentes soluções alcalinas após 28 dias de cura.

\begin{tabular}{llllll}
\hline Amostra & Si[\%at $]$ & Al[\%at] & Na[\%at $]$ & Si/Al[\%at $]$ & Na/Al[\%at $]$ \\
\hline
\end{tabular}




\begin{tabular}{|c|c|c|c|c|c|}
\hline $\begin{array}{l}\text { Seja seu próprio } \\
\text { diferencial } \\
\partial x\end{array}$ & & & & & $\begin{array}{l}\text { UFSJ } \\
\begin{array}{c}\text { Universidade Federal } \\
\text { de São João del-Rei }\end{array}\end{array}$ \\
\hline H12S0-28-90 & 48,79 & 23,34 & 27,86 & 2,09 & 1,19 \\
\hline H16S0-28-90 & 52,64 & 23,82 & 23,54 & 2,21 & 0,99 \\
\hline H12S10-28-90 & 54,59 & 27,9 & 17,51 & 1,96 & 0,63 \\
\hline H16S10-28-90 & 54,29 & 28,7 & 17,01 & 1,89 & 0,59 \\
\hline H12S20-28-90 & 59,3 & 20,96 & 19,74 & 2,83 & 0,94 \\
\hline H16S20-28-90 & 60,33 & 22,11 & 17,55 & 2,73 & 0,79 \\
\hline H12S30-28-90 & 59,13 & 22,7 & 18,17 & 2,60 & 0,80 \\
\hline H16S30-28-90 & 52,88 & 25,1 & 22,02 & 2,11 & 0,88 \\
\hline
\end{tabular}
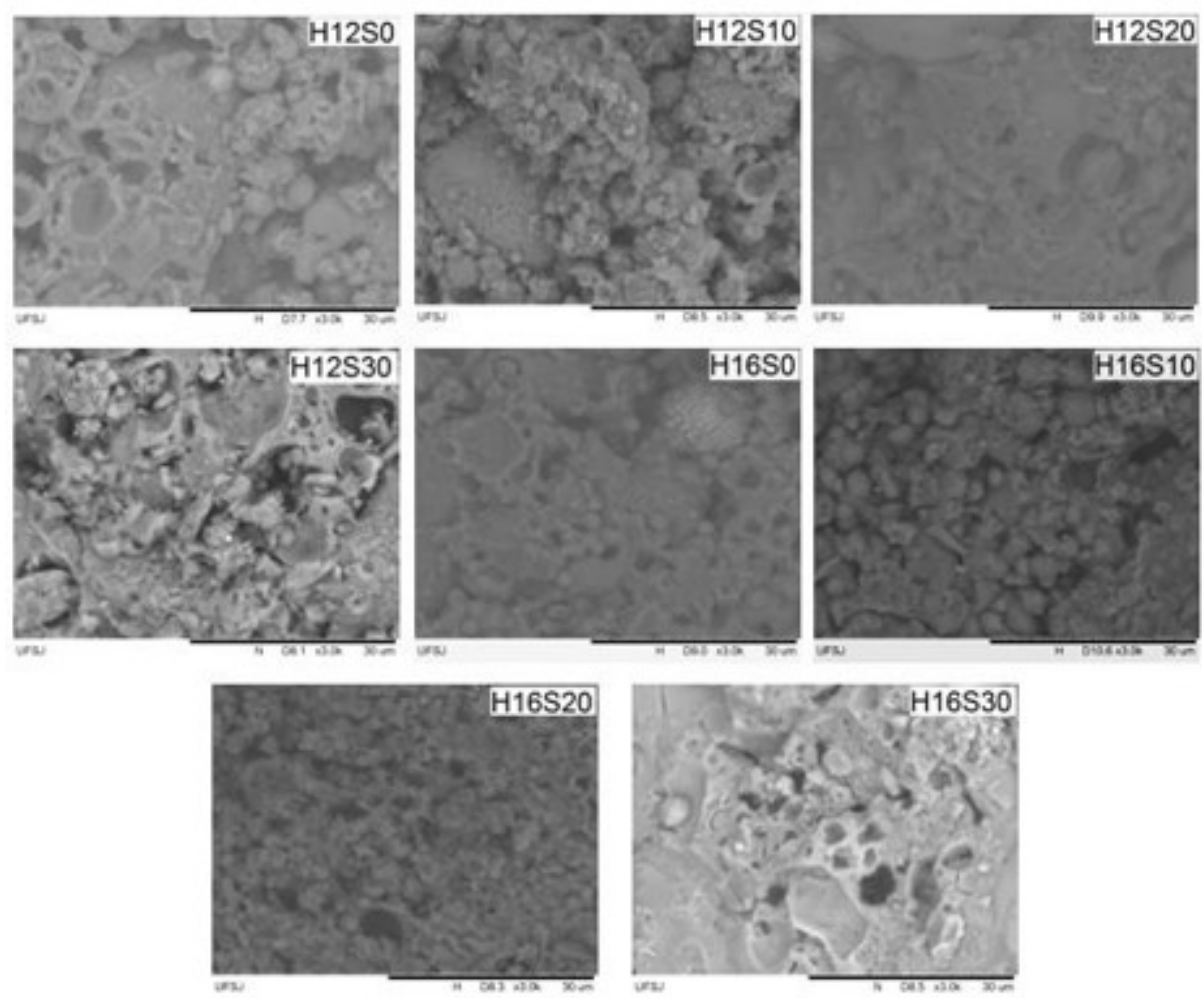

Figura 4. Microscopia eletrônica de varredura dos geopolímeros a base de cinza volante após 28 dias de cura.

\section{CONCLUSÕES}

Os resultados obtidos demonstram que a ativação alcalina das cinzas volantes promoveu a obtenção de matrizes cimentícias com elevada resistência mecânica. As amostram apresentaram resistências próximas de $88 \mathrm{MPa}$ nas primeiras 24 horas de cura. Por meio das análises microestruturais é possível observar que o processo de geopolimerização acarreta em modificações das fases cristalinas presentes em todas as amostras. A adição de $\mathrm{Na}_{2} \mathrm{SiO}_{3}$ nas soluções favoreceu a obtenção de matrizes com baixa absorção de água e porosidade aparente. Os geopolímeros apresentaram propriedades desejáveis para serem usados como candidatos a substituir o cimento 


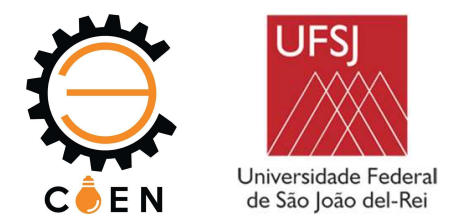

Portland na indústria da construção civil, representando, consequentemente, uma diminuição nos impactos ambientais ocasionados por tal setor industrial.

\section{AGRADECIMENTOS}

Os autores agradecem à Universidade Federal de São João del-Rei (UFSJ), CAPES e CNPQ.

\section{DIREITOS AUTORAIS}

Os autores são os únicos responsáveis pelo conteúdo das informações contidas neste artigo

\section{REFERÊNCIAS BIBLIOGRÁFICAS}

ABNT. NBR - 5739. Concreto - Ensaio de compressão de Corpos-de-prova cilíndricos. (2007).

ASTM. Standard Specification for Coal Fly Ash and Raw or Calcined Natural Pozzolan for Use. Annual Book of ASTM Standards. 3-6, (2010).

ATIŞ, C.D. et al. Very high strength (120MPa) class F fly ash geopolymer mortar activated at different $\mathrm{NaOH}$ amount, heat curing temperature and heat curing duration. Construction and Building Materials v. 96, p. 673678 , out. 2015.

AZEVEDO, A. G. de S. et al. Production of fly ash-based geopolymers using activator solutions with different $\mathrm{Na} 2 \mathrm{O}$ and $\mathrm{Na} 2 \mathrm{SiO} 3$ compositions. Cerâmica v. 63, n. 366, p. 143-151 , 2017.

AZEVEDO, A G S; STRECKER, K. Influência da Composição Química das Soluções Alcalinas Ativadoras na Produção de Geopolímeros a Base de Cinza Volante. Revista Eletrônica de Materiais e Processos v. 12, n. 1, p. 39-46, 2017.

AZEVEDO, Adriano Galvão Souza; STRECKER, Kurt. Brazilian fly ash based inorganic polymers production using different alkali activator solutions. Ceramics International v. 43, n. 12, p. 9012-9018, ago. 2017.

BAKHAREV, T. Geopolymeric materials prepared using Class F fly ash and elevated temperature curing. Cement and Concrete Research v. 35, n. 6, p. 1224-1232, 2005.

BARBOSA, V. F F; MACKENZIE, K. J D; THAUMATURGO, Clelio. Synthesis and

characterisation of materials based on inorganic polymers of alumina and silica: Sodium polysialate polymers. International Journal of Inorganic Materials v. 2, n. 4, p. 309-317, 2000.

BITENCOURT, C S et al. A geopolimerização como técnica para a aplicação do resíduo de bauxita ( Geopolymerization as a technique for bauxite residue applications ). v. 58, p. 20-28, 2012.

CRIADO, M. et al. Effect of the $\mathrm{SiO} 2 / \mathrm{Na} 2 \mathrm{O}$ ratio on the alkali activation of fly ash. Part II: 29Si MAS-NMR Survey. Microporous and Mesoporous Materials v. 109, n. 1-3, p. 525-534, 2008.1387- 1811.

CRIADO, M.; PALOMO, A.; FERNÁNDEZ-JIMÉNEZ, A. Alkali activation of fly ashes. Part 1: Effect of curing conditions on the carbonation of the reaction products. Fuel v. 84, n. 16, p. 2048-2054, 2005.

DAVIDOVITS, J. Geopolymers - Inorganic polymeric new materials. Journal of Thermal Analysis v. 37, n. 8, p. 1633-1656, 1991.

DAVIDOVITS, Joseph. Geopolymers of the First Generation: SILIFACE-Process. Geopolymer '88 v. 1, p. 49-67, 1988.

DAVIDOVITS, Joseph; SAWYER, J.L. Early High-Strength Mineral Polymer . Estados Unidos. 1985.

FERNÁNDEZ-JIMÉNEZ, A.; PALOMO, A. Mid-infrared spectroscopic studies of alkali-activated fly ash structure. Microporous and Mesoporous Materials v. 86, n. 1-3, p. 207-214 , 2005.1387-1811.

IX COEN - Congresso de Engenharias da UFSJ Interconexão. 

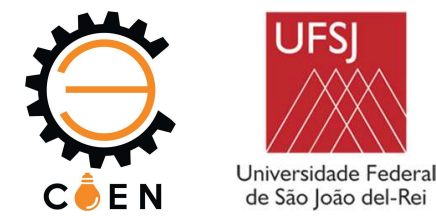

GÖRHAN, Gökhan; KÜRKLÜ, Gökhan. The influence of the NaOH solution on the properties of the fly ashbased geopolymer mortar cured at different temperatures. Composites Part B: Engineering v. 58, p. 371-377 , 2014.

GUO, Xiaolu; SHI, Huisheng; DICK, Warren a. Compressive strength and microstructural characteristics of class C fly ash geopolymer. Cement and Concrete Composites v. 32, n. 2, p. 142- 147, 2010.

MIYAKE, E.T., Determinação da permeabilidade a gás em concreto usado em repositórios para rejeitos radioativos, Instituto de pesquisas energéticas e nucleares, (1994).

MOZGAWA, W.; DEJA, J. Spectroscopic studies of alkaline activated slag geopolymers. Journal of Molecular Structure v. 924-926, p. 434-441, abr. 2009.

NATH, S. K. et al. Microstructural and morphological evolution of fly ash based geopolymers. Construction and Building Materials v. 111, p. 758-765, 2016.

PACHECO-TORGAL, F. et al. Are geopolymers more suitable than Portland cement to produce high volume recycled aggregates HPC. Construction and Building Materials v. 36, p. 1048-1052 , 2012.

PACHECO-TORGAL, Fernando; CASTRO-GOMES, João; JALALI, Said. Investigations about the effect of aggregates on strength and microstructure of geopolymeric mine waste mud binders. Cement and Concrete Research v. 37, n. 6, p. 933-941, 2007.

PROVIS, John L.; DEVENTER, Van; S.J., Jannie. Geopolymers. Structures, Processing, Properties and Industrial Applications. 2009. 464 RAHIER, H.; MELE, B.; WASTIELS, J. Low-temperature synthesized aluminosilicate glasses. Journal of Materials Science v. 31, n. 1, p. 80-85, 1996.

ŠKVÁRA, František; JÍLEK, Tomás; KOPECKÝ, Lubomír. Geopolymer materials based on fly ash. Ceramics - Silikaty v. 49, n. 3, p. 195-204, 2005.

WILLIAMS, Ross P.; VAN RIESSEN, Arie. Determination of the reactive component of fly ashes for geopolymer production using XRF and XRD. Fuel v. 89, n. 12, p. 3683-3692, 2010.

YAHYA, Zarina et al. Comparative study on early strength of sodium hydroxide $(\mathrm{NaOH})$ activated fly ash based geopolymer. AIP Conference Proceedings v. 1887, 2017.

ZAHARAKI, D.; KOMNITSAS, K.; PERDIKATSIS, V. Use of analytical techniques for identification of inorganic polymer gel composition. Journal of Materials Science v. 45, n. 10, p. 2715-2724

\section{FLY ASH BASED GEOPOLYMER PRODCUTION USING SIMPLE AND COMPOUND ACTIVATOR SOLUTIONS}

Adriano Galvão Souza Azevedo (1) (adrianogalvao26@yahoo.com.br),

Luis Fernando Tonholo Domingos (2) (luistonholo@gmail.com), Kurt Strecker (3) (strecker@ufsj.edu.br)

(1) Universidade Federal de São João del Rei (UFSJ) - DCNat - Praça Dom Helvécio, 74 - Fábricas - São João del Rei - MG

(2) Universidade Federal de São João del Rei (UFSJ) - DCNat - Praça Dom Helvécio, 74 - Fábricas - São João del Rei - MG

(3) Universidade Federal de São João del Rei (UFSJ) - DEMEC - Praça Frei Orlando, 170 - Centro - São João del Rei - MG

ABSTRACT: The civil construction is a sector that promotes great degradation of the environment. The geopolymers are a new class of binder material able to be used as a substituent to ordinary Portland cement. The aim of this work was to evaluate the properties of fly ash based geopolymers. The fly ash based geopolymeric samples were produced by activation using different alkaline solutions. The results showed that the activation using simple alkaline solution (only $\mathrm{NaOH}$ ) promotes a high mechanical strength in the first 24 hours (88.0 MPa). The use of sodium silicate (compound solution $\mathrm{NaOH}+\mathrm{Na2SiO3)}$ promotes a gain of 


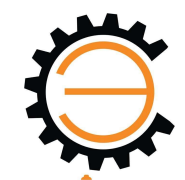

C $\mathrm{EN}$

\section{UFS]}

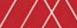

Universidade Federa de São João del-Rei

mechanical strength close to 220\% in some samples. After 28 days of cure, the samples produced with compound activator solutions showed a compressive strength of $50.8 \mathrm{MPa}$ and $3.36 \%$ of porosity. The $x$-ray diffraction and the infrared spectroscopy showed the presence the crystalline phases and the shift of band of $\mathrm{Si}-\mathrm{O}-\mathrm{Si}$ existing in the original fly ash before the activation, respectively. The scanning electron microscopy showed that original spherical morphology of the fly ash was modified to a densified and compacted surface due the formation of aluminosilicate that increase the mechanical strength.

KEYWORDS: Geopolymers, geopolymeric cement, fly ash, alkaline activation 